\title{
PSEUDOREGULAR RADICAL CLASSES
}

\section{N.R. MCConnell and T. Stokes}

\begin{abstract}
We consider radical classes specified by an associating polynomial in two variables which have a similar form to the polynomial defining the class of quasiregular rings. In particular, the relationships of these classes to the classes of semiprime, nil and quasiregular rings are explored.
\end{abstract}

Throughout, all rings are associative. The ring of integers will be denoted by $\mathbf{Z}$. We denote by $\circ$ the circle composition operation on any ring $R$, defined by $a \circ b=a+b-a b$ for all $a, b \in R$.

Let $\mathbf{Z}_{0}[x, y, z]$ be the free ring (that is, the ring of integer polynomials without constant term) on the generators $x, y, z$; similarly, let $\mathbf{Z}_{0}[x, y]$ be the free ring on the generators $x, y$. We view the latter as a subring of the former. An element $f$ of $\mathbf{Z}_{0}[x, y]$ is said to be associating if there exists $g \in \mathbf{Z}_{0}[x, y, z]$ such that $f(f(x, y), z)=f(x, g(x, y, z))$. This notion is defined in much greater generality in [2], for instance by allowing more than one "existential" variable $y$, or by allowing the expression $f$ to be drawn from a set so that the nil radical can be dealt with (amongst others).

For any $f \in \mathbf{Z}_{0}[x, y]$, let $\mathcal{R}_{f}$ be the class of rings in $\mathcal{V}$ defined as follows: $R$ is in $\mathcal{R}_{f}$ providing that, for every $r \in R$ there exists $s \in R$ such that $f(r, s)=0$. By [2, Theorem 4], $\mathcal{R}_{f}$ is a radical class providing $f$ is associating.

The classes of quasiregular and von Neumann regular rings have the form $\mathcal{R}_{f}$ for some $f$ : in the former case, we may let $f=x+y+x y$ and $g=y+z+y z$; in the latter, $f=x-x y x$ and $g=y+z-2 x y z+x y z y x$. Indeed these examples may be generalised as follows: if $p, q \in \mathbf{Z}[x]$, then the element $x-p(x) y q(x)$ of $\mathbf{Z}_{0}[x, y]$ is associating, as is implicitly shown in [5], and the resulting radical class is the class of $(p ; q)$-regular rings. This family also includes Divinsky's D-regular radical class.

\section{Pseudoregularity}

In this paper we consider the special case in which $f(x, y)=p(x)+q(x) y$, where we allow $q(x) \in \mathbf{Z}[x]$; thus we assume $f(x, y)$ is (right) linear in $y$. (There is obviously a dual theory for $f(x, y)=p(x)+y q(x)$.) We use the notation $\mathcal{R}_{p, q}$ as an alternative to $\mathcal{R}_{f}$.

Received 19th February, 2001

Copyright Clearance Centre, Inc. Serial-fee code: 0004-9727/01 \$A2.00+0.00. 
We briefly put this choice in context. It is easy to show there are no associating $f(x, y)$ of the form $f(x, y)=p_{0}(x)+p_{1}(x) y+p_{2}(x) y^{2}+\cdots$ except those for which $p_{i}(x)=0$ for all $i>1$. On the other hand, if we assume commutativity, the family $f_{m}(x, y)=$ $x \circ y \circ y \circ \cdots \circ y$ ( $m$ times) for any square-free $m$ are associating and give rise to the distinct radical classes $\mathcal{J}_{m}=\mathcal{R}_{f_{m}}$ as in [3]. (There are other similar associating $f$ defined in terms of $\circ$, but all give rise to one of these classes.) We know of no other commutative cases. Returning to the non-commutative case, there may perhaps be cases in which $y$ occurs non-linearly in one or more monomials in $f(x, y)$, although we conjecture there are none. Thus it is quite possible that our apparently restrictive assumption on the form of $f(x, y)$ is not very restrictive at all and only rules out of consideration cases where $y$ makes a single appearance in any one monomial.

It will be convenient to say that $(p, q) \in \mathbf{Z}[x] \times \mathbf{Z}[x]$ is an $\mathcal{R}$-pair if $p(0)=0$ and $f(x, y)=p(x)+q(x) y$ is associating. Whether or not $f(x, y)$ is associating, we call the condition $f(x, y)=0$ the $(p \mid q)$-pseudoregularity condition. (Generally, any pair of polynomials written in the form $(p, q)$ will be assumed to satisfy $p(0)=0$.)

It turns out that even in the general non-commutative case, whether $(p(x), q(x))$ is an $\mathcal{R}$-pair depends on single variable, and hence commutative, conditions on $p, q$.

THEOREM 1.1. The pair $(p(x), q(x))$ is an $\mathcal{R}$-pair if and only if

1. $p(p(x))-p(x) \in(q(x))$ and

2. $q(p(x)) \in(q(x))$,

where $(q(x))$ is the principal ideal generated by $q(x)$ in $\mathbf{Z}[x]$.

PROOF: First suppose that $f(x, y)=p(x)+q(x) y$ is associating. Then in $\mathbf{Z}_{0}[x, y, z]$, $f(f(x, y), z)=f(x, g)$ for some $g$, so

$$
p(p(x)+q(x) y)+q(p(x)+q(x) y) z=p(x)+q(x) g(x, y, z) .
$$

Letting $g(x, y, z)=s(x, y)+t(x, y) z$, we have that

$$
p(p(x)+q(x) y)+q(p(x)+q(x) y) z=p(x)+q(x) s(x, y)+q(x) t(x, y) z .
$$

Hence $p(p(x)+q(x) y)=p(x)+q(x) s(x, y)$ and $q(p(x)+q(x) y)=q(x) t(x, y)$ in $\mathrm{Z}[x, y]$. Letting $y=0$ gives the identities $p(p(x))=p(x)+q(x) s(x, 0)$ and $q(p(x))=q(x) t(x, 0)$, holding in $\mathbf{Z}[x]$, and so $p(p(x))-p(x) \in(q(x))$ and $q(p(x)) \in(q(x))$ in $\mathbf{Z}[x]$.

Conversely, if $p(p(x))-p(x) \in(q(x))$ and $q(p(x)) \in(q(x))$ in $\mathbf{Z}[x]$, then we can write $p(p(x))=p(x)+r(x) q(x)$ and $q(p(x))=s(x) q(x)$ for some $r, s \in \mathbf{Z}[x]$. Hence

$$
\begin{aligned}
f(f(x, y), z) & =p(p(x)+q(x) y)+q(p(x)+q(x) y) z \\
& =p(p(x))+q(x) h(x, y)+q(p(x)) z+q(x) k(x, y) z
\end{aligned}
$$


for some $h \in \mathbf{Z}_{0}[x, y], k \in \mathbf{Z}[x, y]$, as can be seen by expanding. Hence

$$
\begin{aligned}
f(f(x, y), z) & =p(x)+q(x) r(x)+q(x) h(x, y)+q(x) s(x) z+q(x) k(x, y) z \\
& =p(x)+q(x)(r(x)+h(x, y)+s(x) z+k(x, y) z) \\
& =p(x)+q(x) g(x, y, z)
\end{aligned}
$$

where $g(x, y, z)=r(x)+h(x, y)+s(x) z+k(x, y) z$, and so $f(x, y)$ is associating.

Further to the earlier comments, there is no obvious way to generalise the form $f(x, y)=p(x)+q(x) y$ and still get simple conditions on $p(x), q(x)$ as above, whether by allowing an $r(x)$ term on the right of the $y$ or even by introducing new existential variables to supplement $y$. Thus, there is a certain inevitability about our choice of form for $f(x, y)$. On the other hand, the conditions are by no means necessary. For instance, the class of Boolean rings $\mathcal{B}$ is $\mathcal{R}_{x^{2}+x, 0}$, and hence is a $(p \mid q)$-pseudoregular radical class, although surely $\left(x^{2}+x, 0\right)$ is not an $\mathcal{R}$-pair; nor does it seem likely that $\mathcal{B}$ is a $(p \mid q)$-pseudoregular class for any $\mathcal{R}$-pair $(p, q)$.

An important special case is $f(x, y)=x+q(x) y$, a radical class for any $q(x) \in \mathbf{Z}[x]$, as is immediate from the above theorem. These are $(q ; 1)$-regular classes. (The discovery of the general $(p ; q)$-regularity condition is attributed to McKnight in [5].) For instance, $f(x, y)=x+y-x y=x+(1-x) y$ has this form, as does $f(x, y)=x+x y$. It is of considerable interest to determine which of our classes are always equal to such classes; that is, which $(p \mid q)$-pseudoregular radical classes are also $(x \mid q)$-pseudoregular (possibly for a different $q$ ). At present we conjecture that if $|q(0)|=1$, then $\mathcal{R}_{p, q}$ is such a case.

PROPOS ITION 1.2. The class $\mathcal{R}_{p, q}$ contains the $(q ; 1)$-regular class $\mathcal{R}_{x, q}$.

Proof: Write $p(x)=x h(x), h(x) \in \mathbf{Z}[x]$. If $R$ is such that for all $a \in R$ there exists $b \in R$ for which $a+q(a) b=0$, then $a=-q(a) b$, so $p(a)=a h(a)=-q(a) b h(-q(a) b)$, so $p(a)+q(a) c=0$, where $c=b h(-q(a) b) \in R$, and so $R \in \mathcal{R}_{p, q}$.

There are a number of easily identified circumstances in which the conditions of Theorem 1.1 are satisfied. Here is one.

Propos ITION 1.3. For any $q(x), s(x) \in \mathbf{Z}[x]$ such that $q(0)=0,(s(x) q(x)$, $q(x))$ is an $\mathcal{R}$-pair.

Proof: If $p(x)$ has the stated form, then, modulo $q(x)$,

$$
p(p(x))-p(x)=s(s(x) q(x)) q(s(x) q(x))-s(x) q(x) \equiv s(0) q(0)=0,
$$

and $q(p(x))=q(s(x) q(x)) \equiv q(0)=0$, as required.

The D-regular radical class defined by $f(x, y)=x+x y$ has this form, with $s(x)=$ 1. If $s(x) \in \mathbf{Z}_{0}[x]$ in the above proposition, then one may let $b=-s(a)$ and then $p(a)+q(a) b=0$ for all $a$ in any ring $R$, so $\mathcal{R}_{p, q}$ is the class of all rings. 
Not all factors $q(x)$ of $p(p(x))-p(x)$ will divide $q(p(x))$. For instance, if $p(x)=$ $x^{2}+2 x$ then $p(p(x))-p(x)=x(x+1)^{2}(x+2)$. Now $q(x)=x, x+1$ both satisfy (2) in Theorem 1.1 (as well as (1) of course), although $q(x)=x+2$ does not. Nonetheless, $q(x)=x(x+2)=p(x)$ does, by Proposition 1.3. In general we have the following, which may be well-known and in a more general form, but we include its proof anyway.

Lemma 1.4. Let $p, q, h \in \mathbf{Z}[x]$. Then letting $d=\operatorname{gcd}(p, q)$ and $r=\operatorname{lcm}(p, q)$, we have that $\operatorname{gcd}(p(h), q(h))=d(h)$, and $\operatorname{lcm}(p(h), q(h))=r(h)$.

Proof: Now we have that $p=d p^{\prime}, q=d q^{\prime}$ for some $p^{\prime}, q^{\prime}$. Then $p(h)=d(h) p^{\prime}(h)$ and $q(h)=d(h) q^{\prime}(h)$, and so $d(h)$ is a common divisor of $p(h), q(h)$. Also, $d=u p+v q$ for some $u, v$, so $d(h)=u(h) p(h)+v(h) q(h)$ and any common divisor of $p(h), q(h)$ is a divisor of $d(h)$. This proves $d(h)=\operatorname{gcd}(p(h), q(h))$. However, we also have that $r=$ $\operatorname{lcm}(p, q)=p q / \operatorname{gcd}(p, q)=p q / d$, and so $\operatorname{lcm}(p(h), q(h))=p(h) q(h) / \operatorname{gcd}(p(h), q(h))=$ $p(h) q(h) / d(h)=r(h)$.

THEOREM 1.5. Suppose $\left(p, q_{1}\right)$ and $\left(p, q_{2}\right)$ are both $\mathcal{R}$-pairs. Then so are $\left(p, \operatorname{gcd}\left(q_{1}, q_{2}\right)\right)$ and $\left(p, \operatorname{lcm}\left(q_{1}, q_{2}\right)\right)$. If $q_{1}(x) q_{2}(x)$ divides $p(p(x))-p(x)$, then $\left(p, q_{1} q_{2}\right)$ is an $\mathcal{R}$-pair.

PROOF: If $q_{1}(x), q_{2}(x)$ both divide $p(p(x))-p(x)$, so $\operatorname{do} q=\operatorname{gcd}\left(q_{1}, q_{2}\right)$ and $r=$ $\operatorname{lcm}\left(q_{1}, q_{2}\right)$. Moreover, $\operatorname{lcm}\left(q_{1}(p), q_{2}(p)\right)=q(p)$. Also, $q_{1}(x)$ divides $q_{1}(p(x))$ and $q_{2}(x)$ divides $q_{2}(p(x))$, so certainly $q=\operatorname{gcd}(q 1, q 2)$ divides $\operatorname{gcd}\left(q_{1}(p), q_{2}(p)\right)=q(p)$, and $r=$ $\operatorname{lcm}\left(q_{1}, q_{2}\right)$ divides $\operatorname{lcm}\left(q_{1}(p(x)), q_{2}(p(x))\right)=r(p(x))$. Hence both $(p, q)$ and $(p, r)$ are $\mathcal{R}$-pairs as required.

Now because $q_{i}(x)$ divides $q_{i}(p(x)), i=1,2$, we have that $q(p(x))=q_{1}(p(x)) q_{2}(p(x))$ is divisible by $q_{1}(x) q_{2}(x)$, so if also $q_{1}(x) q_{2}(x)$ divides $p(p(x))-p(x)$, then $\left(p, q_{1} q_{2}\right)$ is an $\mathcal{R}$-pair.

Suppose $q(x)=r(p(x))$ divides $p(p(x))-p(x)$. Then $q(p(x))=r(p(p(x))) \equiv$ $r(p(x))=q(x) \equiv 0($ modulo $q(x))$, so $(p(x), r(p(x)))$ is an $\mathcal{R}$-pair. There are certain factors of $p(p(x))-p(x)$ which have this form and hence always form $\mathcal{R}$-pairs with $p(x)$.

In general, for $p(x)$ satisfying $p(0)=0$, we have that $p(x)=x h(x)$ where $h(x)=$ $p(x) / x \in \mathbf{Z}[x]$, so

$$
\begin{aligned}
p(p(x))-p(x) & =x h(x) h(x h(x))-x h(x) \\
& =x h(x)(h(x h(x))-1) \\
& =p(x)(h(p(x))-1),
\end{aligned}
$$

of which three obvious factors are $p(x)$ itself, $h(p(x))-1$, and their product $p(p(x))-p(x)$ itself. Thus $q(x)=r(p(x))$ where $r(x)=x, r(x)=h(x)-1$, or $r(x)=p(x)-x$, is a factor of $p(p(x))-p(x)$. Summing up, we have 
Propos ITION 1.6. Given $p(x)$ with $p(0)=0$, the following are $\mathcal{R}$-pairs:
1. $(p(x), p(x))$,
2. $(p(x), p(p(x))-p(x))$, and
3. $(p(x), p(p(x)) / p(x)-1)$.

\section{SEMIPRIME CLASSES}

A radical class is semiprime if it contains the prime (Baer) radical class. Semiprime $(p ; q)$-regular classes turn out to be of special importance, and are characterised by the property that $|p(0)|=|q(0)|=1$. In fact, the semiprime $(p ; q)$-regular radical class is equal to the semiprime $(p q ; 1)$-regular class by $[4$, Corollary 16$]$, in turn equal to the $(x \mid p q)$-pseudoregular class.

Thus an $(x \mid q)$-pseudoregular class (which is always radical, being a $(q ; 1)$-regular radical class) is semiprime if and only if $|q(0)|=1$. 'On the other hand, there are pseudoregular classes $\mathcal{R}_{p, q}$ with $|q(0)|=1$ which are not even radical classes. For instance, $(p, q)=(2 x, 1-x)$ is easily seen not to be an $\mathcal{R}$-pair, but does not even define a radical class. To see this, first note that no ring with identity of characteristic other than 2 is in $\mathcal{R}_{p, q}$, so in particular $R=\mathbf{Z}_{\mathbf{4}} \notin \mathcal{R}_{p, q}$. However, the ideal $I=\{0,2\} \cong \mathbf{Z}_{2}^{0} \in \mathcal{R}_{p, q}$ (where $M^{0}$ denotes the zeroring on the Abelian group $M$ ), and $R / I \cong \mathbf{Z}_{2} \in \mathcal{R}_{p, q}$. Hence $\mathcal{R}_{p, q}$ is not closed under extensions. It is possible that the third class of examples in Proposition 1.6 will provide examples of $(p \mid q)$-pseudoregular radical classes for which $|q(0)|=1$ yet which is not a $(p ; q)$-regular class for any $p, q$. For instance, putting $p(x)=x^{2}$ gives the radical class $\mathcal{R}_{x^{2}, x^{3}-1}$, which may or may not be a $(p ; q)$-regular class for any $p, q$.

A radical class is semiprime if and only if it contains all zerorings ([5, Lemma 3]). Quite generally we have

THEOREM 2.1. $\mathcal{R}_{p, q}$ contains all zerorings if and only if the coefficient of $x$ in $p(x)$ is a multiple of $q(0)$.

Proof: Let $p(x)=a_{1} x+a_{2} x^{2}+\cdots$ and $q(x)=b_{0}+b_{1} x+\cdots$. Now suppose $q(0)=$ $b_{0} \neq 0$. Let $R$ be the zeroring on $\mathbf{Z}_{b_{0}}$, the additive cyclic group of integers modulo $b_{0}$. If $\mathcal{R}_{p, q}$ contains all zerorings, then for all $r \in R$ there exists $s \in S$ for which $a_{1} r+b_{0} s=0$, that is, $a_{1} r=0$ for all $r \in R$, and so $b_{0}$ divides $a_{1}$. Conversely, if $a_{1}=b_{0} c$ for some integer $c$, then for any zeroring $R$, if $r \in R$, then $p(r)=a_{1} r=c b_{0} r=b_{0}(c r)=\operatorname{crq}(r)$, and so $R \in \mathcal{R}_{p, q}$.

Now suppose $b_{0}=0$. Then for any zeroring $R$ and any $r, s \in R, p(r)+q(r) s=a_{1} r$, so if $a_{1}=0$ then $\mathcal{R}_{p, q}$ contains all zerorings. Conversely, if $\mathcal{R}_{p, q}$ contains all zerorings, then letting $R$ be the zeroring on $\mathrm{Z}$, we see that $R \in \mathcal{R}_{p, q}$ implies $a_{1}=0$.

COROLlary 2.2. If $(p, q)$ is an $\mathcal{R}$-pair, then $\mathcal{R}_{p, q}$ is semiprime if and only if the coefficient of $x$ in $p(x)$ is a multiple of $q(0)$. 
From the earlier comments, this actually generalises the corresponding fact for $(p ; q)$ regular classes.

All semiprime $(p ; q)$-regular classes contain $\mathcal{J}$, the Jacobson radical. Here is an example to show that in general a semiprime pseudoregular radical class need not. Let $f(x, y)=x^{2}+\left(x^{4}-x^{2}\right) y$, so that $p(x)=x^{2}$ and $q(x)=x^{4}-x^{2} ;$ the conditions of Theorem 1.1 are satisfied as is easily checked, so $\mathcal{R}_{f}$ is a radical class. It is easy to see that all zerorings are in $\mathcal{R}_{f}$, which is therefore semiprime, so if it were $(p ; q)$-regular for some $p, q$, then it would contain $\mathcal{J}$ (by [4, Theorem 3]). However, $R=\mathrm{Z}_{0}[X] /\left(X^{3}\right)$ is nil and not in $\mathcal{R}_{f}$, since (writing $\bar{X}$ for the image of $X$ in $R$ ), there would need to be a $P(\bar{X}) \in R$ such that $\bar{X}^{2}-\bar{X}^{2} P(\bar{X})=0$, which there is not.

For $(p ; q)$-regularity, semiprimeness is equivalent to $|p(0) q(0)|=1$. If this latter condition is satisfied, then from [1, Corollary 7] and Proposition $1.2, \mathcal{J} \subseteq \mathcal{R}_{x, q} \subseteq \mathcal{R}_{p, q}$. Hence we have

PROPOSITION 2.3. If $|q(0)|=1$ then all quasiregular rings are in $\mathcal{R}_{p, q}$.

\section{Equivalent pairs}

We say $(p, q)$ and $\left(p^{\prime}, q^{\prime}\right)$ are equivalent if $\mathcal{R}_{p, q}=\mathcal{R}_{p^{\prime}, q^{\prime}}$. In general we have the following

PROPOSITION 3.1. If $(p, q)$ is an $\mathcal{R}$-pair then so is $(p+s q, q)$ for any $s$ for which $s(0) q(0)=0$. If $s(0)=0$ then $(p, q)$ and $(p+s q, q)$ are equivalent.

PRoOF: If $(p, q)$ is an $\mathcal{R}$-pair, then letting $p^{\prime}=p+s q$, we see that $p^{\prime}(0)=0$, and that modulo $q(x), p^{\prime}(x) \equiv p(x)$, so

$$
\begin{aligned}
p^{\prime}\left(p^{\prime}(x)\right)-p^{\prime}(x) & \equiv p^{\prime}(p(x))-p(x) \\
& =p(p(x))+s(p(x)) q(p(x))-p(x) \\
& \equiv p(p(x))-p(x) \\
& \equiv 0
\end{aligned}
$$

and $q\left(p^{\prime}(x)\right) \equiv q(p(x)) \equiv 0$, so $\left(p^{\prime}, q\right)$ is an $\mathcal{R}$-pair.

Now note that $p^{\prime}(x)+q(x) y=p(x)+q(x)(s(x)+y)$, so if $s(0)=0$ then evidently $\mathcal{R}_{p, q}$ is equivalent to $\mathcal{R}_{p^{\prime}, q}$.

We say $(p, q)$ and $\left(p^{\prime}, q^{\prime}\right)$ are strongly equivalent if $q=q^{\prime}$ and $p-p^{\prime} \in(x q(x))$, the ideal generated by $x q(x)$ in $\mathbf{Z}[x]$. Notation: $(p, q) \Leftrightarrow\left(p^{\prime}, q^{\prime}\right)$. From the last proposition, strong equivalence of $(p, q)$ and $\left(p^{\prime}, q^{\prime}\right)$ implies their equivalence, and each or neither is an $\mathcal{R}$-pair. Equivalence does not imply strong equivalence: for instance, all $(p, 1)$ are equivalent, since all define the (radical) class of all rings.

PROPOSITION 3.2. If $q(x)$ has leading coefficient of magnitude 1 , then $(p, q)$ is strongly equivalent to $(r, q)$, where $r(x)$ is the remainder of $p(x)$ on division by $x q(x)$. 
Proof: We can divide $p(x)$ by $x q(x)$ since the latter has leading coefficient of size 1 , in such a way that the remainder $r(x)$, congruent to $p(x)$ modulo $x q(x)$, has degree at most that of $q(x)$. Moreover $(r, q)$ is strongly equivalent to $(p, q)$ by Proposition 3.1.

\section{SOME SPECIAL CASES}

THEOREM 4.1. For $n>0$, the expression $f(x, y)=x^{n}+\left(x^{m}-x^{l}\right) y$ is associating if and only if $m, l \leqslant n^{2}$ and $m-l$ divides $n^{2}-n$.

Proof: We want $q(x)=x^{m}-x^{l}$ to divide $q(p(x))=x^{n m}-x^{n l}$, which it surely does as is easily seen from working modulo $q(x)$, but also to divide $p(p(x))-p(x)=x^{n^{2}}-x^{n}$. For this to occur it must be the case that $m-l$ can divide $n^{2}-n$ but also that $m, l \leqslant n^{2}$.

For example, from this we obtain that $f(x, y)=x^{n+1}+x^{n} y-x^{n+1} y=x^{n}(x \circ y)$ is associating. $\mathcal{R}_{f}$ defines a class which obviously contains $\mathcal{J}$. However, $x^{n+1}+\left(x^{n}-\right.$ $\left.x^{n+1}\right) y=x^{n+1}+(1-x) z$ where $z=x^{n} y$, so $\mathcal{R}_{x^{n+1}+\left(x^{n}-x^{n+1}\right) y} \subseteq \mathcal{R}_{x^{n+1}+(1-x) y}$; but $x^{n+1}+(1-x) y$ defines the same class as $x^{n+1}+(1-x)\left(x+x^{2}+\cdots+x^{n}\right)+(1-x) y=$ $x+(1-x) y=x \circ y$, so $\mathcal{R}_{x^{n}(x \circ y)}=\mathcal{J}$, for all $n \geqslant 0$. A great many radical $\mathcal{R}_{p, q}$ turn out to be $\mathcal{J}$ as is evident from results in [4]: if $|q(0)|=1$ then this is the case exactly when $q(x)$ has a factor of the form $(a x+1)$, where $a \neq 0$ and for each prime divisor $m$ of $a$, there is an integer $n$ such that $m$ divides $p(n)$.

THEOREM 4.2. Suppose $(p, q)$ is an $\mathcal{R}$-pair. If $q(1)=0$ and $p(1)=1$ then $\mathcal{R}_{p, q} \subseteq \mathcal{J}$.

ProOF: Now if $q(1)=0$ then $q(x)=(x-1) h(x)$ for some $h(x)$. If in the ring $R$, $p(a)+(a-1) h(a) b=0$, then $p(a)+(a-1) c=0$ for $c=h(a) b \in R$, so $\mathcal{R}_{p, q} \subseteq \mathcal{R}_{p, x-1}$. Now if $(p, q)$ is an $\mathcal{R}$-pair, then $p(p(x))-p(x)$ is divisible by $q(x)$ and hence certainly by $x-1$, and moreover $p(x)-1$ is divisible by $x-1$ exactly when $p(1)=1$, so that in this case, $(p(x), x-1)$ is an $\mathcal{R}$-pair. But as can be seen by computing $p(x)$ modulo $x(x-1)=x^{2}-x,(p(x), x-1) \Leftrightarrow(p(1) x, x-1)=(x, x-1)$, which defines $\mathcal{J}$.

Letting $p(x)=q(x)=x$ gives the D-regular radical, which is surely not in $\mathcal{J}$ since it contains all rings with identity, while $p(x)=q(x)=x^{2}-x$ gives a still smaller radical class, although one which still contains all rings with identity and hence is not in $\mathcal{J}$ either. These provide examples to show that both conditions in the above theorem statement are necessary.

Combining Theorem 4.2 with Proposition 2.3 gives

CoROLlary 4.3. If $(p, q)$ is an $\mathcal{R}$-pair with $p(1)=1, q(1)=0$ and $|q(0)|=1$ then $\mathcal{R}_{p, q}=\mathcal{J}$.

The requirement that $|q(0)|=1$ in this corollary cannot be dropped, as the fact that $\mathcal{J} \neq \mathcal{R}_{x^{2}, x^{4}-x^{2}}$ shows. 
Corollary 4.4. If $p(1)=1$, then $(p(x), 1-p(x))$ is an $\mathcal{R}$-pair, that is $p(x) \circ y$ is associating, and moreover $\mathcal{R}_{p, 1-p}=\mathcal{J}$.

This result is rather surprising; it implies for instance that for a ring to be quasiregular, it is sufficient for all $n$-th powers to be quasiregular, for any single $n>0$. On the other hand, the following result holds quite generally, and follows easily from Proposition 3.1 .

Proposition 4.5. For any $t(x) \in \mathbf{Z}[X]$, the class of rings defined by $f(x, y)=$ $x(t(x) \circ y)$ is a radical class.

Next we consider which pseudoregular classes contain all nil rings.

THEOREM 4.6. Suppose $p(x)=a_{s} x^{s}+a_{s+1} x^{s+1}+\cdots$ and $q(x)=b_{t} x^{t}+b_{t+1} x^{t+1}+$ ... with $a_{s}, b_{t} \neq 0$. If the class $\mathcal{R}_{p, q}$ contains all nil rings then $s>t$, and if also $b_{t}$ divides all other $b_{j}$, then $b_{t}$ divides all $a_{i}$.

Conversely, if $s>t$ and $b_{t}$ divides all other $a_{i}$ as well as all $b_{j}$, then $\mathcal{R}_{p, q}$ contains all nil rings.

Proof: Suppose first that $\mathcal{R}_{p, q}$ contains all nil rings. Suppose $s \leqslant t$. Let $R=$ $\mathbf{Z}_{0}[X] /\left(X^{t+1}\right)$. Then $R$ is nil and so for the image $\bar{X}$ of $X$ in $R$, there is some $h \in R$ for which $a_{s} \bar{X}^{s}+a_{s+1} \bar{X}^{s+1}+\cdots+a_{t} \bar{X}^{t}=\left(b_{t} \bar{X}^{t}\right) h$, which is zero since $h$ is a multiple of $\bar{X}$; hence $a_{s}=a_{s+1}=\cdots=a_{t}=0$, a contradiction, so $s>t$. Hence we can write $p(x)=a_{t+1} x^{t+1}+a_{t+2} x^{t+2}+\cdots$, where all $a_{i}=0$ for $i<s$ (if any such $i \geqslant t+1$ exist).

Now assume all $b_{j}$ are divisible by $b_{t}$. Let $R=Z_{b_{t}}^{0}[X] /\left(X^{m}\right)$, the ring of polynomials over $\mathbf{Z}_{b_{t}}$ with zero constant term factored by the ideal $\left(X^{m}\right)$, where $m$ exceeds the maximal degrees of $x$ in either $p(x)$ or $q(x)$. Then $R$ is nil, so, again letting $\bar{X}$ be the image of $X$ in $R$, there is $h \in R$ for which $a_{t+1} \bar{X}^{t+1}+a_{t+2} \bar{X}^{t+2}+\cdots=0$ in $R$, since all $b_{j}$ are divisible by $b_{t}$. Hence $a_{t+1}, a_{t+2}, \ldots$ are divisible by $b_{t}$ also.

Now suppose conversely that $b_{t}$ divides all $b_{j}$ and all $a_{i}$. Let $b_{i}^{\prime}=b_{i} / b_{t}$ and $a_{i}^{\prime}=a_{i} / b_{t}$ for all $i>t$. Then let $r(x)=b_{t+1}^{\prime} x+b_{t+2}^{\prime} x^{2}+\cdots$ and $w(x)=a_{s}^{\prime} x_{s-t+1}+a_{s+1}^{\prime} x_{s-t+2}+\cdots$; then $p(x)=b_{t} x^{t} w(x)$ and $q(x)=b_{t} x^{t}(1-r(x))$. Suppose $R$ is nil, with $a \in R$ such that $a^{n}=0$. Let $b=-w(a)\left(1+r(a)+r(a)^{2}+\cdots+r(a)^{n-1}\right)$, noting that $r(a)^{n}=0$. Then

$$
\begin{aligned}
& p(a)+q(a) b \\
& =b_{t} a^{t} w(a)-b_{t} a^{t}(1-r(a)) w(a)\left(1+r(a)+r(a)^{2}+\cdots+r(a)^{n-1}\right) \\
& =b_{t} a^{t} w(a)\left(1-(1-r(a))\left(1+r(a)+r(a)^{2}+\cdots+r(a)^{n-1}\right)\right) \\
& =b_{t} a^{t} w(a)\left(1-\left(1+r(a)+r(a)^{2}+\cdots+r(a)^{n-1}-r(a)-r(a)^{2}-\cdots-r(a)^{n}\right)\right) \\
& =b_{t} a^{t} w(a)(1-1) \\
& =0 .
\end{aligned}
$$

Hence $R \in \mathcal{R}_{p, q}$. 
This has immediate application to cases where $(p, q)$ is an $\mathcal{R}$-pair, in terms of radical classes containing the nil radical class. It would be nice to tighten the above result to if and only if status, dropping the need to assume $b_{t}$ divides all other $b_{j}$ in the first part of the statement. There is also the question of the uniqueness of the element $b$ given $a$ in the nil ring $R$, satisfying $p(a)+q(a) b=0$, a question of interest for pseudoregular rings generally. Both of these issues may require the assumption that $(p, q)$ is an $\mathcal{R}$-pair in order to make progress.

It is easy to give sufficient conditions for hereditariness of $\mathcal{R}_{f}$. These are the relevant version of conditions given in $[\mathbf{5}]$, which they generalise in the $(q ; 1)$-regular case.

PROPOSITION 4.7. $\mathcal{R}_{f}$ is hereditary providing either of the following holds:

1. $|q(0)|=1$; or

2. $q(x)=p(x) s(x), s(0)=0$.

Proof: If $|q(0)|=1$ then $q(x)=1+x s(x)$ or $-1+x s(x)$ for some $s(x) \in \mathbf{Z}[x]$. Hence in the first case, $f(x, y)=p(x)+q(x) y=p(x)+y+x s(x) y$, so if $R \in \mathcal{R}_{f}$, with $I$ an ideal of $R$, and if $a \in I$ then $p(a)+b+a s(a) b=0$ for some $b \in R$, so $b=-p(a)-a s(a) b \in I$. Similarly with the second case.

Suppose $q(x)=p(x) s(x), s(0)=0$. Let $R \in \mathcal{R}_{f}, f(x)=p(x)+q(x) y$, with $I$ an ideal of $R$. If $a \in I$ then there exists $b \in R$ for which $0=p(a)+q(a) b=p(a)+p(a) s(a) b=$ $p(a)+s(a) p(a) b=p(a)+s(a)(-q(a) b) b=p(a)+q(a)\left(-s(a) b^{2}\right)=p(a)+q(a) c$, where $c=-s(a) b^{2} \in I$. Hence $I \in \mathcal{R}_{f}$ and so $\mathcal{R}_{f}$ is hereditary.

It is not known if a converse for this result can be obtained.

\section{REFERENCES}

[1] T.L. Goulding and A.H. Ortiz, 'Structure of semiprime ( $p ; q)$-radicals', Pacific J. Math. 37 (1971), 97-99.

[2] N.R. McConnell and T. Stokes, 'Equationally defined radical classes', Bull. Austal. Math. Soc. 47 (1993), 217-220.

[3] N.R. McConnell and T. Stokes, 'Radical classes of commutative rings defined in terms of the adjoint operation', Comm. Algebra 22 (1994), 5533-5548.

[4] J.D. McKnight Jr. and G.L. Musser, 'Special ( $p$; $q$ )-radicals', Canad. J. Math. 24 (1972), $38-44$.

[5] G.L. Musser, 'Linear semiprime ( $p$; $q)$ radicals', Pacific J. Math. 37 (1971), 749-757.

Department of Defence

Locked Bag 5076

Kingston ACT 2604

Australia
School of Mathematical and Physical Sciences Murdoch University

Murdoch WA 6150

Australia 